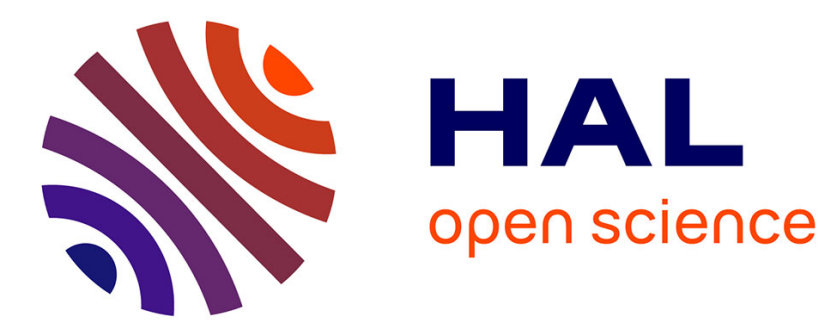

\title{
La disqualification sociale des éleveurs intégrés
}

Christian Nicourt, Jacques Cabaret

\section{To cite this version:}

Christian Nicourt, Jacques Cabaret. La disqualification sociale des éleveurs intégrés. Revue d'Etudes en Agriculture et Environnement - Review of agricultural and environmental studies, 2014, 95 (2), pp.225-251. 10.4074/S1966960714012041 . hal-01189995

\section{HAL Id: hal-01189995 \\ https://hal.science/hal-01189995}

Submitted on 1 Sep 2015

HAL is a multi-disciplinary open access archive for the deposit and dissemination of scientific research documents, whether they are published or not. The documents may come from teaching and research institutions in France or abroad, or from public or private research centers.
L'archive ouverte pluridisciplinaire HAL, est destinée au dépôt et à la diffusion de documents scientifiques de niveau recherche, publiés ou non, émanant des établissements d'enseignement et de recherche français ou étrangers, des laboratoires publics ou privés. 


\title{
La disqualification sociale des éleveurs intégrés.
}

\author{
Christian NICOURT*, Jacques CABARET** \\ * INRA, UR1216 RiTME, F-94205 Ivry, France \\ e-mail: Christian.Nicourt@ivry.inra.fr \\ ** INRA, UR1282 ISP, F-37380 Nouzilly, France
}

Résumé - L'objectif de cet article est de s'interroger sur le travail et le métier qu'exercent les éleveurs intégrés. Nous soutenons l'hypothèse que l'intégration disqualifie socialement le travail des éleveurs qui la pratiquent voire contribue au déni de leur métier. En effet, les voies de leur professionnalisation les désignent - aux yeux des autres agriculteurs et notamment de leurs intégrateurs - comme des déchus ou des précarisés de la production agricole d'un côté et, de l'autre, comme statutairement dépendants d'autres éleveurs. Dès lors, nous nous proposons d'éclairer cette disqualification sociale et ce déni à partir d'entretiens compréhensifs auprès d'éleveurs intégrés et d'éleveurs intégrateurs. L'article examine d'abord les parcours professionnels qui conduisent les éleveurs à l'intégration. Il analyse ensuite leurs manières singulières de travailler et d'exercer leur métier. Enfin, il caractérise la disqualification du travail et le déni du métier qui les affectent.

Mots-clés : travail, métier, intégration, identité, éleveur, élevage, agriculture

\section{The social disqualification of subcontracting breeders}

Abstract - This study explored whether subcontracted breeders are socially disqualified in their profession by their own views or by those they believe to be expressed by the traditional breeders. The elements of social disqualification include social fragility (how they are perceived by the majority of conventional producers) and economic dependency on integrators who provide the selected animals, food and even therapeutics in some cases. While subcontracting breeding is largely regarded as a more economical means to produce livestock or poultry, this study hypothesized the subcontractors are socially disqualified on the grounds of having minimal professional autonomy or knowledge on the actual breeding or management of livestock. Comprehensive interviews were conducted with subcontracting breeders of calf, pig and poultry. We show that social disqualification of subcontractors is based on their feeling of over-dependence on the farm integrator and the integrators lack of recognition for their quality of work.

Keywords: labour, profession, subcontracting, stockbreeder, livestock, agriculture

Classification JEL: J29, J70 


\section{Introduction}

Ce sont des entretiens récents avec des éleveurs de volaille bio en intégration des Côtes-d'Armor et de la Sarthe qui nous ont conduits à nous réinterroger sur l'identité professionnelle des éleveurs intégrés. L'intégration de tels éleveurs apparaît de prime abord surprenante, vu l'importance que les bio accordent à la notion d'autonomie, qui consisterait autant à construire des manières endogènes de travailler reliées à leur situation, qu'à produire l'alimentation de leurs animaux et assumer leur mise en marché. Pour eux, cette capacité à se gouverner et agir par soi-même (Ehrenberg, 2010) est une condition de la bio. Surprise aussi qu'ils s'organisent entre eux et discutent leurs méthodes d'élevage avec les techniciens de leurs groupements intégrateurs. Surprise enfin des difficultés de certains à débattre au sein du $G^{1} B^{1}$ avec leurs collègues, qui leur dénient leur qualité de bio, parce qu'intégrés.

S'interroger sur le travail et l'identité professionnelle des éleveurs inscrits dans une relation contractuelle n'est pas aisé. Cette relation recouvre des conditions et des dénominations multiples, dont les significations peuvent varier, au point que certains éleveurs ne savent pas toujours comment qualifier leur situation, ni même parfois comprendre la nature des relations qu'ils entretiennent avec leurs contractants (Diry, 1985). Néanmoins, plutôt que la notion d'économie contractuelle, avancée par Jollivet et al. (1971), qui permet de s'interroger aujourd'hui aussi sur le statut de nombre d'éleveurs contemporains qui se considèrent "indépendants ", nous adopterons celle d'intégration, plus restreinte ; surtout aussi parce que c'est celle qu'utilisent la plupart des éleveurs impliqués. Ainsi qualifierons nous ce vaste ensemble d'éleveurs qui se considèrent et sont reconnus par leurs pairs comme tels : ces autres éleveurs qui partagent les mêmes manières d'exercer leur travail et de le concevoir, les mêmes expériences et épreuves dans l'accomplissement de leur métier. Un ensemble qui prendrait en compte le façonnier, dépendant étroitement de son éleveur-intégrateur, jusqu'à l'éleveur achetant ses intrants - aliments, engrais . . - - et revendant ses animaux à une même entreprise, dont les techniciens influencent les manières de travailler, de façon plus ou moins relâchée.

Dans la perspective de Davezies (1993), l'identité au travail de tels éleveurs est le fruit d'un double jugement : être éleveur intégré est un construit identitaire, au croisement du regard des pairs et de celui de ceux qui n'ont pas une expérience sensible de ce travail, mais en jugent les produits : intégrateurs, clients, marchands, techniciens, éleveurs non intégrés ... Alors, si l'on considère que le métier est le fruit d'une spécialisation délimitant continûment des frontières et excluant ses marges pour affirmer son autonomie (Latreille, 1979), l'éleveur intégré est, comme bien d'autres, du côté des exclus. Il est autant vulnérable économiquement que socialement (Castel, 1991). Il est de ceux dont le travail est disqualifié et le métier dénié ; une telle occultation

\footnotetext{
${ }^{1}$ Groupement des Agriculteurs Biologiques.
} 
de la réalité de l'engagement professionnel fragilise l'identité (Dejours, 2000). Le déni permet la mise à distance voire à l'écart de phénomènes ou de populations, comme c'est le cas de celles qui exercent un métier socialement repoussant ou suspect : charbonniers à l'écart dans les bois, vidangeurs ... (Corbin, 1982). Aussi, notre objectif est-il d'éclairer la disqualification du travail et le déni du métier de ces éleveurs, qui leur fait acquérir et intérioriser un statut dévalorisé (Paugam, 1991), en nous interrogeant sur leurs parcours professionnels et leurs manières d'exercer leur métier.

L'élevage en intégration apparaît en France au milieu des années 1950. Cette notion floue et polysémique, que les agriculteurs emploient pour désigner les relations contractuelles qu'entretiennent certains d'entre eux avec des entreprises d'amont et/ou d'aval, est d'abord à l'initiative de l'Etat, dont les agents de la régie des droits, au ministère des finances, encadrent au plus près ceux qui cultivent du tabac depuis 1810 : comptant les plants autorisés, arrachant les surnuméraires, jugeant et classant les qualités ... Ensuite, l'intégration mise en place par les coopératives viticoles, notamment en Languedoc-Roussilllon depuis le début des années 1900, semble peu peser sur les viticulteurs. Ce n'est en effet que tout récemment, avec la mise en place de cahiers des charges "qualitatifs ", que les techniciens des coopératives effectuent un cadrage et un contrôle soutenus de leur travail (Nicourt et Girault, 2011). Dans l'après-guerre, des agriculteurs de grandes cultures, qui avaient déjà une longue expérience préalable des contrats avec des sucreries, contractent avec des conserveurs de légumes. De tels contractants ont des situations économiques qui leur permettent de négocier en quelque sorte d'un pied d'égalité avec leurs intégrateurs (Rémy, 1971). Les relations contractuelles ne sont en effet pas toutes empreintes de rapports de domination asymétriques. Puis ce sont les industriels de l'alimentation animale qui, à la fin des années cinquante, entraînent des éleveurs dans l'agriculture contractuelle : d'abord la volaille, puis les porcs et les veaux. Dès lors les marques de domination des intégrateurs sur les éleveurs semblent plus affirmées. L'intégration reprend ici le modèle technique américain des feedlots, ces unités d'engraissement de bovins, annexées à des exploitations de polyculture élevage dans les zones de la corn-belt, consacrées à la production intensive du maïs, mises en place dès les années 1920 (Coléou, 1997). Au sein de ce mouvement, il faut aussi souligner la présence des tenants de l'agriculture biologique que furent les adhérents de Lemaire-Boucher dès 1962. Ceux-ci devaient en effet acheter à la firme du lithothamne - une algue calcifiée réputée engrais naturel universel - et une variété de semence pour qu'elle commercialise leur blé. Le développement de cet ensemble d'agriculture contractuelle, lors des années soixante, nourrit aussi bien les débats qu'organisent les élites syndicales agricoles, ainsi un numéro dédié de la Revue Paysan (1961), que le questionnement des chercheurs, notamment ceux du Groupe de Sociologie Rurale (Bodiguel et al., 1971). Les premiers s'insurgent contre le risque de prolétarisation des éleveurs en y voyant le passage « du paysan souverainement indépendant à l'ouvrier étroitement dirigé ». Estrangin (1962), dans une 
parution ultérieure de la revue, souligne d'ailleurs qu'aux États-Unis de tels éleveurs de porcs auraient adhéré à un syndicat ouvrier. Certes, au congrès du Centre National des Jeunes Agriculteurs (CNJA) de 1964, les syndicalistes mettent de l'eau dans leur vin : le rapport Liaudon (1964) propose en effet une «agriculture de groupe industrielle et commerciale ", en transformant les exploitations « en ateliers spécialisés et de les rattacher à des ensembles plus vastes, soit au niveau de la production, soit en amont, soit en aval [...] dans des groupes d'une dimension et d'une structure appropriée ». L'intégration se métamorphose alors, aux yeux des syndicalistes, en exigence économique ; ainsi devient-elle acceptable, lorsqu'elle se réalise sous l'égide de la «profession organisée ». Pourtant, il ne semble pas que les gestionnaires coopératifs soient plus tendres avec ceux qu'ils intègrent. C'est ce que souligne Burguière (1975), décrivant une coopérative qui "propose au paysan une intégration moins barbare, mais non moins stricte que l'intégration capitaliste ». Sa description ne fait que confirmer les réserves émises précédemment par Malassis (1964), dubitatif quant à la capacité des coopératives à mettre en place des relations avec les agriculteurs, autres que celles des firmes privées. En revanche, les sociologues s'accordent pour considérer l'intégration comme un recours potentiel pour les agriculteurs en situation professionnelle précaire. Ainsi pour Lamarche (1971) : «ce sont bien souvent de "petits" exploitants agricoles, dont l'exploitation n'est guère viable ", sachant que l'intégration ne fournit alors pour beaucoup qu'un répit pour des contractants déjà précarisés (Jollivet et al., 1971).

Ce qui peut être qualifié aujourd'hui d'intégration s'inscrit aussi dans une histoire plus ancienne encore : celle du travail à façon et de la sous-traitance industrielle (Houssiaux, 1957, 1) comme de la pluriactivité. Cet exercice simultané ou successif par une même personne de plusieurs activités professionnelles différentes (Cornu, 1987) a particulièrement concerné les ménages agricoles. Dès le XIX ${ }^{\mathrm{e}}$ siècle, les agriculteurs - hommes et femmes, et souvent leurs enfants - constituent en effet une main-d'œuvre de choix pour la proto-industrialisation en milieu rural, surtout dans les domaines du textile et de la petite métallurgie (Lequin, 1977), lorsque triomphe «l'établi associé à l'étable » (Mayaud, 1999).

Aujourd'hui, l'élevage en intégration concerne essentiellement la volaille, le porc et le veau. En volaille, $70 \%$ de la production est intégrée ; elle implique environ 10000 éleveurs de poulet de chair finis en 2008 ; 95,8\% de ceux qui élèvent du poulet «standard » sont en intégration au moins partielle, de même que 98,1\% de ceux qui élèvent du poulet « label » (Callais et Pendaries, 2010). D'après le Recensement Agricole de 2010, $34 \%$ des porcs sont engraissés à façon. Cela concerne environ 9500 exploitations, soit environ $40 \%$ de celles qui élèvent des porcs. Quant aux éleveurs de veaux « de boucherie », la plupart sont intégrés.

Nous avancerons l'hypothèse que l'intégration disqualifie socialement les éleveurs qui la pratiquent voire contribue au déni de leur métier, 
parce que les voies de leur professionnalisation les désignent - aux yeux des autres agriculteurs et notamment de leurs intégrateurs - comme des déchus ou des précarisés de la production agricole d'un côté et qu'ils sont statutairement dépendants d'autres éleveurs, de l'autre. Notre propos repose essentiellement sur la reprise d'entretiens compréhensifs (Hughes, 1996), menés auprès d'éleveurs intégrés depuis près d'une trentaine d'années : plus d'une vingtaine en volaille, bio et non bio, autant en porcs, partagés entre intégrés et intégrateurs, et moins d'une dizaine en veaux, en Bretagne, Sarthe et Dordogne. Nous examinerons d'abord qui sont les éleveurs intégrés et les voies de leur professionnalisation. Nous analyserons ensuite l'originalité de leurs manières d'exercer leur métier. Dès lors nous nous interrogerons sur la disqualification de leurs manières de travailler et le déni professionnel dont ils sont l'objet.

\section{Les voies de la professionnalisation des éleveurs intégrés}

Les éleveurs intégrés sont surtout ceux qui sont condamnés à disparaître par les organismes du développement, du fait de leur trop faible surface ou des limites de leurs capacités économiques. Une situation qui ne permet ni de faire de l'élevage extensif, ni de disposer des ressources financières et techniques nécessaires pour accéder aux méthodes d'intensification, préconisées par les organismes du développement. Ces éleveurs se retournent alors vers les intégrateurs qui leur proposent de mettre en place des élevages de volailles, de porcs ou de veaux hors-sol. L'intégration permet aussi le maintien d'agriculteurs double actifs. C'est ce que souligne Philippe Lacombe (1968), notamment à propos du secteur avicole. Alors, d'où viennent ces éleveurs et qu'attendent-ils de l'intégration ? Comment leur professionnalisation dessine-t-elle des orientations qui se diversifient sur la durée, dans la perspective des sentiers de dépendance (Dobry, 2000)?

\subsection{Une diversité des parcours des éleveurs intégrés}

La rapidité du démarrage de l'élevage en intégration a correspondu à la conjonction de la revendication d'autonomie dans le travail des jeunes agriculteurs et du souci de leurs parents de les installer à moindre frais. L'usage de bâtiments d'élevage en hors-sol, a renforcé la décohabitation du travail entre les générations et a accompagné celle de leurs résidences. De même qu'elle a conforté la spécialisation, notamment en dissociant l'élevage laitier et celui de volaille ou de porcs.

L'intégration ... Ça correspondait à une attente économique parce qu'il y avait besoin de faire du revenu. Ça correspondait à une attente des jeunes qui voulaient s'installer et puis il n'y avait pas forcément de place sur l'exploitation. En plus ça évitait d'avoir un conflit 
intergénérationnel. Donc le mec, le père lui disait : tu fais une porcherie dans ce coin-là, tu te débrouilles. Et globalement c'était bien vécu. (Un éleveur de porcs intégrateur des Côtes d'Armor).

Une telle installation n'est cependant possible que pour les élevages laitiers qui disposent ou peuvent trouver assez de terres pour y établir une porcherie fonctionnant en hors-sol. Colson (1980) montre que ces jeunes éleveurs de porcs qui s'installent sont de ceux dont les parents - éleveurs laitiers - ont assez d'aisance pour susciter chez eux une volonté de poursuite du métier d'éleveur. Pour des agriculteurs moins dotés, l'intégration peut aussi être une voie de promotion. En volaille les premiers éleveurs intégrés - plutôt aisés et considérés comme modernistes - ont suscité l'engagement d'autres, plus modestes, qui ainsi se sont vus requalifiés face aux non contractants devenus de nouveaux «attardés» (Lamarche, 1971). En dépit des parcours différents qui les ont conduits à l'intégration, les éleveurs qui ont opté pour cette manière d'élever ont en partage une incapacité à s'installer sans avoir recours à de tels contrats.

La plupart des éleveurs intégrés ont débuté leur métier dans des conditions précaires. Une précarité liée essentiellement au fait qu'ils disposaient d'exploitations économiquement fragiles. Tous ont commencé à exercer leur métier sur de "petites exploitations ", c'est-à-dire considérées comme inférieures à l'exploitation « moyenne » de leur territoire d'implantation, selon le regard des autres agriculteurs comme de celui des techniciens agricoles. L'intégration n'implique en effet ni qu'ils cultivent l'alimentation de leurs animaux, et donc disposent des surfaces nécessaires, ni qu'ils doivent l'acheter. Les premiers contrats qu'effectuent les éleveurs intégrés ne les obligent également pas à des investissements onéreux, en leur permettant de réutiliser leurs vieux bâtiments. Pour ceux qui peuvent construire eux-mêmes leurs bâtiments, c'est alors un moyen de se développer grâce aux premiers gains réalisés.

En 67-68, on a fait du porc. On faisait que l'engraissement. On a démarré dans l'appentis en bas, petit. Et on a fini dans la grande porcherie au-dessus. Je crois que c'est 200 porcs qu'on avait. Chaque année, on faisait un bâtiment. (une éleveuse intégrée de Dordogne).

Les intégrateurs fournissent l'alimentation des animaux, les reprennent lorsqu'ils sont «engraissés » et rémunèrent l'agriculteur en fonction du poids acquis par les animaux et de l'alimentation consommée : «l'indice ». L'éleveur touche ainsi un revenu qui peut être quasi stable, semblable à un salaire. Certains intégrateurs de même cautionnent auprès des banques la construction de bâtiments pour les éleveurs avec lesquels ils contractent. C'est ainsi que pour les néo-ruraux - du moins ceux qui n'ont pas la possibilité d'écouler leurs 
produits en circuits courts - l'intégration est une des voies privilégiées pour entrer dans le métier.

Elle était aux PTT et moi chauffeur de bus à la RATP. On est venus ici en 82 . On a trouvé une ferme où il y avait des vieux bâtiments de poulets et on s'est dit : pourquoi pas se lancer dans l'agriculture. Comme on n'avait aucun diplôme d'agriculture, je suis retourné faire un BEPA pour nous installer ... Ici, il y avait environ 12 hectares à vendre, donc on a acheté. On était en poulets Label. En 89, on s'est installé. Elle, elle a choisi la production de porc Label Rouge. On avait déjà trois bâtiments de poulets, tout en intégration. On est passés en bio en 96. (Un couple d'éleveurs néo-ruraux de poulets bio intégrés des Côtes d'Armor).

Les néo-ruraux recherchent aussi dans l'intégration un guidage dans le métier. C'est pour eux un mode d'apprentissage. En effet, les éleveurs intégrés sont encadrés par des techniciens, qui sont autant là pour leur prescrire les manières de travailler de l'intégrateur, que pour contrôler leur travail. Certains intégrateurs proposent également des réunions techniques. Chez tous, le cahier des charges, qui vise à orienter le travail de l'éleveur pour qu'il réponde aux exigences de l'intégrateur, introduit, de fait, une programmation de son travail. Il implique l'usage de techniques et la transcription sur papier de certaines opérations effectuées. Une telle écriture est pour ces éleveurs une ressource et une mémoire de leur travail (Joly, 2009). Cependant, elle a toujours un statut ambivalent ; d'un côté elle guide le travail, de l'autre, c'est une obligation destinée à être contrôlée par un prescripteur.

Les éleveurs intégrés sont aussi, surtout en porc, de ceux qui ont fait faillite. Trop vulnérables pour survivre professionnellement dans un univers marqué par la concurrence entre éleveurs et les conséquences de la variation des cours du marché du porc, l'intégration est pour eux autant un moyen de conserver l'héritage familial de la ferme que de continuer à exercer le métier, avec un statut qui leur permet de retrouver une sécurité financière. Certes, la grande majorité des éleveurs intégrés a démarré son parcours professionnel en situation précaire. En revanche, l'intégration les a installés dans le métier, soulignant la diversité des voies qu'ils ont empruntées.

\subsection{Les choix contraints des éleveurs intégrés}

Au début des années 1970 , les éleveurs intégrés sont près de six sur dix à juger que le travail sous contrat leur est favorable (Rambaud, 1973). Ces " petits exploitants », que les tenants de l'agriculture de groupe appellent à disparâtre, constituent alors une population importante. Pour ceux qui ont une carence d'expérience agricole, l'accompagnement de techniciens de 
la société intégratrice est un atout recherché pour démarrer dans le métier. Les mobiles qui les conduisent à l'intégration divergent selon leurs situations et leurs projets. Pour certains, c'est l'opportunité de valoriser d'anciens bâtiments ; pour d'autres c'est un travail peu prenant qui permet d'exercer une seconde activité ; pour d'autres enfin, c'est une forme nouvelle de polyculture-élevage qu'ils recherchent.

quelle que soit leur situation initiale, petits éleveurs, néo-ruraux, jeunes installés, c'est la sécurité financière que leur assure l'intégration que mettent en avant les éleveurs pour expliquer leur choix. Cette sécurité financière peut recouvrer différentes significations. Pour l'un, c'est l'assurance d'un apport régulier qui permet de faire face aux échéances des emprunts. Pour l'autre, c'est se garantir face aux variations de cours ou au faible rapport d'une production gratifiante sur le plan identitaire. Ce revenu d'assurance, que certains comparent à « la paye du lait » ou au «salaire d'un fonctionnaire », s'inscrit dans une constance des revendications de l'ensemble des agriculteurs : recherche de prix garantis, d'un revenu régulier, de primes compensatoires ...

L'élevage intégré est le plus souvent lié à d'autres productions. C'est pour certains éleveurs une nouvelle manière d'associer élevage laitier et porcin aux cultures. L'organisation du travail aussi bien que les objectifs agronomiques se complètent. Tandis que nombre d'intégrateurs porcins cherchent à se débarrasser de leurs surplus de lisier - car les textes réglementaires imposent de les traiter ou de rechercher des surfaces d'épandage adéquates, pour limiter la teneur en nitrates des eaux - c'est pour les éleveurs laitiers presque une aubaine que d'économiser ainsi de l'engrais minéral.

Il y a énormément de porcheries d'engraissement qui sont à côté d'un atelier lait. Les deux vont bien ensemble ... . Et en plus, au niveau pollution, c'est le top. Tu fous un lisier sur une prairie, t'as aucun ruissellement, tu as l'utilisation qui est au maximum. C'est vrai que ça colle bien. Pour faire du maïs, tu mets un petit peu de fumier, un petit peu de lisier, ça marche du top. C'est un complément de revenu et t'as le lisier en plus. (un éleveur de porcs intégré des Côtes d'Armor).

L'intégration est considérée comme une opportunité de survie par certains éleveurs tels ceux qui ne faisaient pas partie de l'aristocratie locale de l'élevage allaitant en Sarthe. Pour ces éleveurs, le travail sous contrat a permis le maintien d'un tissu social agricole. Partout, ils s'accordent pour considérer que l'engraissement réclame peu de travail et offre de la souplesse et de la disponibilité pour d'autres productions. C'est ainsi que certains l'ont utilisé comme appoint périodique pour compenser les aléas de leur production principale. Pour tous, c'est une production secondaire. C'est aussi parce que le travail qu'elle exige est considéré comme négligeable. 
En engraissement, il n'y a pas beaucoup de soin. Bon, ils sont supplémentés au départ dans l'aliment ou dans l'eau ... Enfin j'aime bien faire un tour, aller voir comment que ça va quand même, surveiller ... C'est pas trop de boulot l'engraissement et en intégration, on n'a pas de risques ... En horaires, c'est assez souple ... Tu peux y aller à 8 heures, à 10 heures du matin. C'est juste pour passer, quoi. (un éleveur intégré des Côtes d'Armor).

Peu d'éleveurs intégrés se passionnent pour un tel travail. Ce qui ne signifie pas qu'ils ne soient pas passionnés d'agriculture. C'est qu'ils exercent leur passion ailleurs dans leur métier. C'est ainsi que pour certains l'intégration assure un revenu qui permet la mise en place progressive d'un autre projet de production. Pour d'autres, elle permet de chercher, d'inventer et de tester d'autres manières de cultiver en bio : association de cultures, nouvelles méthodes de travail du sol ... Face au cadrage de l'intégration, ces éleveurs cherchent à exercer leur normativité (Canguilhem, 1966), c'est-à-dire à construire leurs propres normes de travail, selon leurs situations. Le plaisir du questionnement et de la construction de ses manières de faire, face à la standardisation et la massification des pratiques de l'intégration, alimente leur quête identitaire. C'est un désir de métier (Osty, 2003) qui passe par leur engagement subjectif dans un tel travail, que manifestent ces éleveurs. Ce désir fonctionne en termes de compensation : ce mécanisme d'auto-défense du moi, qui permet de contrebalancer une insatisfaction ressentie dans un autre domaine.

En poulet, à telle date il faut faire le vaccin, à telle date on change d'aliment, ainsi de suite. Donc, c'est calé, il n'y a pas de recherche particulière à faire. En culture, c'est une recherche permanente. Et puis chaque agriculteur a sa petite recette. Il n'y en a pas beaucoup qui font pareil, donc c'est là que c'est intéressant ... Dans les cultures, tout dépend de moi. Chaque décision, c'est moi qui la prends et personne d'autre : qu'est-ce que je vais mettre dans ce champ-là ? Quelle variété ? Quel assolement ? Quel travail du sol ? Il n'y a rien d'écrit. Donc, c'est à moi de prendre la décision à chaque fois. Si un jour, j'ai plus le piment de chercher, ça aura beaucoup moins d'intérêt. (Un éleveur de poulet bio intégré de Sarthe).

Pour les intégrateurs, le risque est grand que les éleveurs négligent les productions intégrées de leurs exploitations ; c'est pourquoi certains multiplient les services. L'un offre un appui technique aux éleveurs, au-delà de la partie intégrée de leur exploitation. Un autre propose des animations techniques sur des sujets choisis par les adhérents des Groupements de 
Producteurs. Un autre encore, pour assurer la fluidité de la production autant que pour se fidéliser les éleveurs, met en place un service de remplacement.

Les projets qui ont conduit les éleveurs à opter pour l'intégration soulignent qu'ils la considèrent comme l'une des rares voies qui puissent leur permettre, à partir de leur situation initiale, de poursuivre le métier d'agriculteur ou d'y entrer. Pour eux, l'intégration est un choix contraint. Mais cette contrainte est d'autant mieux assumée qu'elle est une voie raisonnable, qui permet d'accéder à la diversité des projets de chacun. Au long de ce parcours, peu délaissent l'intégration. Chacun garde au moins une production sous contrat : «en assurance ». Celle qui ne l'est pas est celle dans laquelle il exerce le projet qui l'a conduit à choisir le métier d'agriculteur. Ainsi l'intégration définit une complexité singulière du métier au sein de laquelle chaque éleveur impliqué considère que c'est grâce à cette manière de le pratiquer, qu'il continue à l'exercer et à y trouver du plaisir, en ayant pu choisir la voie qui lui convient. L'intégration permet ainsi autant l'entrée dans le métier que de s'y maintenir, malgré des situations défavorables, au regard des critères professionnels institués.

\section{L'originalité des manières de faire des éleveurs intégrés}

On peut s'interroger sur l'objectif de sécurité financière que mettent en avant les éleveurs qui ont opté pour l'intégration. En effet, celle-ci est une relation de travail identique à la sous-traitance. Les contrats y sont des accords déséquilibrés en faveur du donneur d'ordre, pouvant aller jusqu'à l'asservissement du contractant (Segrestin, 1996). L'éleveur intégré, à défaut d'être asservi, est pour le moins dépendant de son fournisseur et client exclusif. C'est un travailleur précaire. Quant à son organisation du travail, soumise aux prescriptions de l'intégrateur, elle souligne les limites de son autonomie au sein de sa propre exploitation. C'est probablement pourquoi certains exercent une activité diversifiée pour sortir de ce cadrage ; mais tous n'ont pas cette latitude.

\subsection{La précarité de l'emploi de l'éleveur intégré}

L'intégration s'inscrit dans la transformation de la relation au marché des éleveurs lors des années soixante. Plus particulièrement pour ces éleveurs, la sécurité acquise en n'étant plus soumis à la variation des cours a pour contrepartie un changement de culture : ne plus se préoccuper ni discuter de la valeur de son produit. L'intégration fait voler en éclat l'implication des éleveurs dans leur travail sur les foires et marchés, cette construction de leur revenu, liée à leurs compétences de vente et plus particulièrement de marchandage, qui mettait en avant la qualité de leur travail d'élevage. Cet abandon remet en cause la reconnaissance publique de telles compétences et l'identité qu'elle conférait à l'éleveur. De même que le pouvoir qu'il semblait 
avoir sur la formation du prix, notamment dans les régions d'embouche du maquignonnage (Wackermann, 1977).

À l'époque, beaucoup dans la région, c'était des petites exploitations qui trafiquaient pas mal, c'était de la main à la main, c'était du liquide. Dès l'instant qu'il y avait un truc intégré, là, il y avait des comptabilités. C'était un gros changement de culture. (un éleveur de poulet bio intégré de Sarthe).

La mise à distance des éleveurs du marché les rend dépendants de leurs intégrateurs. Pour eux, le contrat d'intégration est conçu comme un emploi : il est dédié à un travailleur ou fournit un revenu complémentaire. Un tel emploi vise à assurer l'équilibre économique de l'exploitation. Cependant, il n'est pas dénué d'incertitudes, susceptibles de fragiliser et de rendre instable la situation des éleveurs; d'autant que le contrat est souvent de courte durée (Schaller, 1979). Depuis la faillite de la Rurale Morbihannaise au début des années soixante, les éleveurs ont appris que la sécurité d'emploi et de revenu qu'ils recherchaient ainsi pouvait être limitée. L'exemple contemporain de la mise en redressement judiciaire du groupe Doux, en Bretagne, confirme ce risque : en situation de récession économique, les donneurs d'ordres se séparent de leurs sous-traitants. Ce fut notamment le cas du secteur automobile qui a adossé la flexibilité de sa production sur leur précarisation (Vennin, 1975). Les éleveurs ont connu des situations semblables, notamment lors des périodes où les intégrateurs se sont concentrés et ont sélectionné leurs éleveurs. Plus communément, les éleveurs qui ne se soumettent pas aux exigences du cahier des charges qui leur est imposé apprennent à leurs dépens le pouvoir des intégrateurs.

Avec le père T. (l'intégrateur), la voisine s'est faite engueuler parce qu'elle faisait son lait sans thermomètre : le sien était cassé. Chez B., le technicien avait demandé de mettre du «Spécial Toux »; elle ne croyait pas à ce médicament, le technicien l'a vu et l'a dit au père $\mathrm{T}$. Ils leur ont repris les silos et ils ont dû chercher ailleurs. Le technicien avait aussi remarqué qu'elle ne soignait pas à heures régulières. (une éleveuse intégrée de Dordogne).

Récemment encore, les éleveurs de porcs intégrés se considéraient en quelque sorte indispensables, du fait des surfaces d'épandage qu'ils offraient à leurs intégrateurs. Ils considéraient même que l'intégration leur servait parfois surtout à gérer leur lisier. Ceux-ci pouvaient ainsi poursuivre leur croissance en «mettant en pension » les animaux qui faisaient dépasser les volumes de lisier qu'ils étaient autorisés à épandre sur leurs exploitations. Cependant, le développement du questionnement environnemental de l'élevage porcin a fragilisé la pérennité de l'emploi des éleveurs intégrés. Aussi se considèrent-ils 
maintenant soumis à la même pression que leurs intégrateurs. Et ils soulignent l'accroissement de leur dépendance en admettant n'avoir aucun poids dans une telle hypothèse.

Le jour où ils décident par exemple de baisser la quantité de porcs produits, qu'est-ce qu'on peut y faire ? Rien. Si jamais ils instaurent des quotas sur les truies par exemple en Bretagne et puis on va abattre $25 \%$, pourquoi pas. Ils pourraient faire ça. Ils pourraient faire un truc comme ça et, nous, on n'a rien à faire. On peut fermer la boutique parce qu'il n'y aura plus de porcs. Pour moi, on n'est pas vraiment acteurs au même titre qu'on l'est dans la filière à lait. Dans la filière à lait, on est responsable, on peut donner une orientation à ce qu'on veut. (un éleveur intégré des Côtes d'Armor).

Lors des dernières années, le rapatriement des porcs « mis en pension » chez des façonniers s'est opéré au fur et à mesure de la concentration des élevages de porcs. Notamment à l'occasion de leur agrandissement lié à leurs mises aux normes - environnement, bien-être - des intégrateurs ont délaissé leurs façonniers. Un tel mouvement est susceptible de s'accélérer. En effet, depuis le décret du 17 janvier 2011 « relatif au regroupement et à la modernisation de certaines installations classées d'élevage ", les éleveurs intégrateurs peuvent regrouper leurs animaux sur leur site initial, avec un effectif qui peut être quasi doublé. Le décret ne les contraint plus à se soumettre à une procédure d'autorisation, longue et porteuse de critiques sociales (Bonnaud et Nicourt, 2006), s'ils se mettent aux normes environnementales, par exemple en augmentant la capacité de leurs stations d'épuration. La possibilité leur est de ce fait offerte de se séparer des éleveurs qu'ils intégraient. Les éleveurs intégrés n'ignorent pas cette situation. Ils savent en effet que leur choix de l'intégration a été contraint. Ils savent aussi qu'il leur a permis de vivre de leur travail, tout en entretenant leur précarité.

\subsection{L’autonomie limitée du travail des éleveurs intégrés}

Les éleveurs intégrés soulèvent le problème des limites de leur autonomie dans leur travail. Certes, il serait naïf de considérer qu'ils appliquent à la lettre les consignes qui leur sont enjointes. D’une part parce que, comme pour les autres travailleurs, elles sont rarement opératoires : en témoignent les grèves du zèle qui paralysent le fonctionnement productif. De même, l'ergonomie de l'activité, fondée sur la remise en cause du paradigme taylorien, a bien montré l'écart entre le travail prescrit et le travail réel, lié à la variabilité des situations de travail, et surtout à l'intelligence de ceux qui travaillent, face aux tâches qui leur sont confiées (de Montmollin, 1983). D'ailleurs, les organisations ont bien compris l'intérêt des prescriptions floues, pour laisser à ceux qui opèrent le 
choix des méthodes les plus appropriées selon les situations (de Terssac, 1992). L'autonomie face aux prescriptions dans le travail ne concerne donc pas que les éleveurs intégrés. En revanche c'est un problème que posent ces éleveurs, dès lors qu'ils se considèrent comme tels. Pour eux, l'absence d'autonomie de décision et d'action les qualifierait, ou plutôt les disqualifierait.

L'éleveur intégré se désigne souvent comme simple exécutant. Tout laisse pourtant à penser qu'il s'engage dans son travail au-delà de la prescription qui lui est enjointe. Certes, il est soumis aux contraintes d'un contrat. Mais celui-ci, de fait, differe peu de ce qui est désigné ailleurs de cahier des charges, pour un éleveur «indépendant ». C'est ainsi qu'un éleveur, au long du même entretien, montre l'importance de l'expérience sanitaire qu'il s'est construite dans son élevage, pour résoudre les problèmes qu'il a rencontrés. Pourtant, son statut dévalorisé le conduit d'abord à occulter son engagement et les compétences qu'il y exerce. Il intériorise sa disqualification en ne mettant pas en avant l'expérience qu'il a acquise, ni les compétences qu'il met en œuvre.

On n'est pas loin de l'intégration parce qu'on ne décide de rien ... Pour moi, il n'y a que la partie financière qui reste ... Il n'y a pas une grosse marge parce qu'on ne décide pas de la date de mise en place; on ne décide pas de ce qu'on va mettre comme espèce. On ne décide pas grand-chose, en fait ... Mon système marche pas trop mal mais parce que j'ai essayé un tas de produits, un tas de trucs, tout ce qui est possible et imaginable ... Je suis obligé de composer à chaque fois, de jouer sur la température, sur l'alimentation, des choses comme ça. Et bon, ça se gère tranquillement. Je me suis fait mon petit protocole à moi. (un éleveur de poulet bio intégré de Sarthe).

Les relations de l'éleveur intégré avec son technicien apparaissent toujours empreintes de tensions. Pour lui, il s'agit de se préserver des marges d'autonomie, face à la standardisation des manières de faire que tente de lui imposer le technicien. L'objet de ces tensions porte souvent sur les soins. Aux prescriptions standardisées des techniciens, les éleveurs mettent en avant leurs compétences acquises. Face à chaque problème rencontré, ils expérimentent. Leur ignorance préalable du travail d'élevage les a conduits en effet à se constituer un ensemble de connaissances, particulièrement adapté aux singularités de leurs élevages. Leurs tâtonnements, érigés en méthode, se sont mués sur la durée en une expérience, qu'ils opposent à celle de leurs techniciens. C'est une sourde confrontation qui émerge alors. Elle leur permet de «tenir » d'un point de vue identitaire, face à certains de leurs prescripteurs, parfois méprisants.

Je pense que bien souvent, c'est des petits trucs qu'il faut essayer. Quand on voit un veau qui ne va pas, il faut essayer ... J'avais dit la dernière fois qu'il faudrait leur 
donner du fer. Ils manquaient d'appétit. Le technicien m'a répondu que l'eau était ferrugineuse, que ce n'était pas la peine. Il a fait une connerie. Ça s'est vu au poids des veaux ... Pour les techniciens, tel médicament, il est pour ça, tel autre ... Moi, pas toujours. Avec la pratique, on acquiert une autre technique ... Les techniciens viennent pour surveiller les veaux et surveiller l'éleveur. C'est eux qui déterminent les médicaments et en même temps, c'est un peu le gendarme. Quand le technicien passe, c'est surtout pour faire des remarques. (une éleveuse de veaux intégrée de Dordogne).

Selon les intégrateurs, l'engraissement de porcs est réputé être un travail aisé, qui mobilise peu l'éleveur. On comprend que celui-ci se défende en expliquant les compétences qu'il exerce pour répondre à la réputation qui lui est faite. Les éleveurs sont en effet prolixes pour caractériser les signes de dégradation de la santé de leurs animaux : oreilles pendantes, yeux tristes, allure dans les déplacements ou anormalité de l'immobilité, comportement de mise à l'écart ... Ils comparent d'une bande à l'autre, mettant en avant leur expérience. Les relations entre éleveur intégré et intégrateur ne sont cependant pas toutes tendues. Lorsque l'intégrateur est un éleveur, chacun a intérêt à échanger avec l'autre pour prévenir et résoudre au mieux les problèmes. Certes, le donneur d'ordre fournit un historique des porcelets qu'il transmet. Mais il prévient aussi de la fragilité d'un lot, en précisant au mieux les symptômes apparus jusqu'à la fin du sevrage, pour que l'éleveur intégré redouble de vigilance. Leurs échanges téléphoniques peuvent être ainsi fréquents en début d'engraissement. Plutôt que le recours à la certification, que mettent en place les donneurs d'ordres de l'industrie et certaines coopératives, la relation entre les partenaires, construite sur la durée et adossée à de multiples échanges, devient empreinte de confiance (Baudry, 1994). C'est notamment le cas lorsque la proximité spatiale inscrit intégrés et intégrateurs dans une histoire commune, au travers de laquelle chacun a appris à connaître les manières de faire de l'autre (Maget, 1955). Alors, la relation établie conforte la situation de chacun : emploi pour l'intégré, réseau d'obligés et notabilité locale pour l'intégrateur. Ailleurs, la distance spatiale entretient la distance sociale.

On supplémente en fonction aussi des difficultés que peut connaître le naisseur pendant le post-sevrage. Il prévient ... Quand ça va mal chez lui, il le dit et quand moi aussi ça ne va pas très bien non plus, je le lui dis aussi. Il passe un coup de fil. On fait tout par téléphone. S’il y a une bande qui est plus fragile sur le plan digestif, il me prévient et moi je fais ma commande d'aliment en fonction. (un éleveur de porcs intégré des Côtes d'Armor). 
La relation entre éleveurs intégrés et intégrateurs est complexe. C'est selon leur parcours et leur situation que les éleveurs intégrés l'apprécient. Face au discours des responsables professionnels qui comparaient l'intégration avec le travail ouvrier, comme archétype d'un travail inscrit dans une relation de dépendance, certains s'inscrivent en porte-à-faux. C'est ce qu'ont considéré les jeunes éleveurs qui se sont affranchis ainsi de la domination paternelle au sein de l'exploitation familiale. C'est aussi le cas de néo-ruraux, pour qui l'intégration a permis d'exercer le métier d'éleveur. Pour les éleveurs précarisés ou déchus, le jugement est plus variable. Cependant, malgré la diversité de leurs parcours et de leurs situations, ce qui organise leur travail est le produit d'une tension constante entre leur dépendance de l'intégrateur et leur quête d'autonomie. Exerçant pour la plupart leur métier en tant que pluriactifs, comment faire reconnaître leur identité d'éleveurs intégrés pour les uns, comment être reconnus pour le métier qu'ils exercent à côté pour d'autres?

\subsection{L'influence de l'intégration sur les manières de travailler des agriculteurs}

Historiquement la complémentarité des productions, en termes de maind'œuvre et d'agronomie, a soutenu le développement de l'intégration. C'est une manière d'exercer leur métier qu'ont choisi, tout au long de leur parcours, certains éleveurs. Ce fut aussi, pour d'autres, permettre le démarrage d'un projet qui ne pourra aboutir qu'à terme. D'autres encore ont diversifié leur activité, lorsque le cadrage de l'intégration leur a pesé. Pourtant, ce sont surtout les agricultrices qui, dans des exploitations familiales, où elles n'ont pas la possibilité d'influencer les orientations, ont été les plus affectées par l'intégration.

L'intégration permet autant de créer un emploi sur une exploitation que d'assurer une complémentarité entre productions. Une telle organisation répond aux besoins de cohérence que recherchent les éleveurs, comme tous les travailleurs (de Keyser et Olivier, 1972). Cette cohérence est assurée également au travers des compétences acquises à la rencontre des prescriptions du technicien intégrateur et de leur pratique. Ils ont ainsi construit une expérience qu'ils peuvent réutiliser d'une production à l'autre. Un tel transfert de compétences non formalisées a été abondamment utilisé dans le monde industriel, pour minorer la qualification des salariés et notamment des femmes (Shaw, 1981). Les compétences des éleveuses sont restées de même invisibles lorsqu'elles les ont transférées d'un élevage à l'autre, d'autant plus pertinemment lorsque les mêmes bâtiments réinvestis soulevaient des problèmes sanitaires identiques. L'intégration leur a permis de réutiliser l'existant : autant les bâtiments vétustes que les compétences acquises. Certains éleveurs et éleveuses ont réalisé ainsi l'ensemble de leur carrière professionnelle, passant de contrat en contrat, au fur et à mesure des 
opportunités. L'intégration est devenue pour eux une manière d'exercer leur métier et d'en embrasser la diversité.

On a fait du poulet, on a fait de la pintade, on a fait de la dinde, enfin c'était dans les mêmes bâtiments ... On faisait porc, volaille et tabac. C'est à cette époque-là qu'on a dû arrêter la vache laitière et qu'on s'est mis sur la Limousine ... Sur les veaux, on a eu l'histoire de la vache folle ... On avait 170 veaux anglais quand la panique est arrivée, ils étaient prêts à partir ... On a diminué les veaux et on s'est mis sur le canard. En intégration aussi. (une éleveuse de veaux intégrée de Dordogne).

Pour ces agriculteurs, l'intégration est devenue une manière de travailler inscrite dans la durée, dont la variation des productions a construit des expériences orientées dans une même perspective, qui ont donné vie au métier. Pour d'autres, l'intégration permet d'entreprendre un projet professionnel à terme. C'est ce qui fait « tenir » l'éleveur. Cette quête à l'issue incertaine est aussi celle d'une réappropriation de leur travail : une recherche d'autonomie et de performance qui est aussi une quête de soi (Ehrenberg, 1991). C'est ici le sens de son travail qui interroge l'éleveur. En s'investissant subjectivement dans une activité complémentaire, il recherche un équilibre entre nécessité économique et plaisir dans le travail. L'intégration, grâce au revenu régulier qu'elle peut procurer, permet en effet d'envisager des projets qui ne pourront aboutir que sur la durée, pour des raisons d'économie ou de construction d'une clientèle. Alors, dans ces exploitations de polyculture-élevage apparaît plus manifeste la distinction des productions au niveau du sens qu'y prend le travail pour l'éleveur. Dans un cas, il s'agit d'assurer un revenu, et l'intérêt pour le métier n'est qu'instrumental. Dans l'autre, il s'agit de contribuer à mener à bien un projet professionnel longuement mûri. L'intégration acquiert alors le statut d'une ressource offrant une souplesse qui en permet l'aboutissement.

J'ai fait une formation à Angers par correspondance, dans le domaine des légumes. En 1983, la ferme s'est agrandie parce qu'il y en avait une qui se libérait à côté et j'ai commencé à faire un peu de porcs. J'avais aussi un peu de poulets. Là-bas, c'était uniquement de l'engraissement à façon avec une coopérative. J'ai arrêté un peu le porc ; j’ai repris. C'était un peu en fonction de comment fonctionnait le tourisme équestre. (Un éleveur néo-rural de poulet bio des Côtes d'Armor).

À l'encontre de ceux qui considèrent que l'intégration bride leur autonomie d'éleveur, elle en est pour d'autres un moyen. Cependant, tout dépend de la situation des éleveurs et de leurs possibilités d'acquérir cette autonomie. 
Certes, dans les exploitations familiales, l'intégration a permis l'autonomie des jeunes hommes en les soustrayant à la dépendance paternelle dans leur travail et en leur offrant une spécialisation, considérée comme une entrée dans la profession. En revanche, une telle spécialisation s'est traduite pour les agricultrices en une organisation singulière de leur travail qui a consolidé leur dépendance. Les hommes apprécient que, temporellement circonscrits tôt le matin et en début de soirée, les soins leur laissent le cœur de la journée disponible, pour réaliser ce qu'ils ont programmé. Tandis que les femmes ont une programmation plus floue, car il leur faut aider, répondre à des aléas et assumer les tâches domestiques (Nicourt et Filippi, 1987). L'organisation du travail qui se met en place avec l'élevage en intégration se distingue ainsi selon le genre de ceux qui interviennent. Face aux exigences "prioritaires " du travail du chef d'exploitation, les agricultrices pallient ses déficiences : le prévenir de l'arrivée d'un technicien, aller chercher une pièce manquante ... Et le remplacer pour soigner ses animaux, lorsque les cultures le maintiennent tardivement aux champs.

Eux, si vous voulez, ils ont leur boulot fixe. Quand il a attaqué de labourer, il labourera du matin jusqu'au soir. Et même il me dira, si à 5 heures tu peux, je ne remonte pas, je ne soigne pas. C'est moi qui soigne. Ils ne sont pas interrompus les hommes (Une éleveuse de veaux intégrée de Dordogne).

Les soins biquotidiens comme la surveillance aléatoire des animaux deviennent souvent le domaine des agricultrices, même lorsque c'est leur conjoint qui est censé l'effectuer. Ce qui conduit d'ailleurs les techniciens des groupements intégrateurs à souligner par leurs propos l'invisibilité et l'absence de reconnaissance du travail de ces femmes. Ils feignent alors d'ignorer la situation et réclament, de manière renouvelée, à s'entretenir avec « le patron » du bâtiment. L'élevage intégré définit une organisation du travail qui peut autant brider les manières de travailler des éleveurs que leur permettre de s'engager subjectivement dans leur travail. Cet engagement apparaît essentiellement lorsqu'ils s'inscrivent dans la perspective d'une polyculture-poly-élevage. Celle-ci permet en effet aux éleveurs d'exercer plusieurs métiers, de distinguer les significations qu'ils donnent à chacun, et de s'engager selon leurs attentes subjectives dans une spécialisation plus que dans un autre. En revanche, l'équilibre auquel aboutit une telle organisation demeure invisible pour autrui. Dès lors domine une identité d'éleveur marqué par une manière d'exercer le métier qui d'un côté ne s'est pas engagé dans la spécialisation et de l'autre a perdu toute autonomie. Deux arguments qui autorisent l'expression de la stigmatisation sociale des éleveurs intégrés. 


\section{Le déni du métier d'éleveur intégré}

C'est dans la relation aux autres, dans la mise en société du travail que se construit l'identité professionnelle. Dans cette perspective, celle des éleveurs intégrés est problématique. Comme celle du sous-traitant industriel, c'est un maillon opacifié du processus de production, escamoté par les producteurs reconnus. L'éleveur intégré apparaît comme un produit inachevé de la spécialisation agricole. Il exerce en quelque sorte un reste de métier, qui aujourd'hui s'agglomérerait avec d'autres scories, pour former une entité désormais considérée comme sans signification. En agriculture, ce serait une résurgence de la "polyculture-élevage ", qu'auraient dû enrayer plusieurs décennies de "développement agricole ». Qu'en est-il alors de l'identité au travail des éleveurs intégrés ? C'est à la rencontre du regard de ces éleveurs et de leurs intégrateurs sur leur travail qu'il est possible de l'apprécier ; le jugement dévalorisé des uns construisant la mésestime de soi des autres. Cette jonction permet le déni du métier : une occultation de son existence, vectrice de souffrance (Dejours, 2000).

\subsection{La dépendance consentie des éleveurs intégrés}

Les éleveurs intégrés considèrent qu'ils exercent un travail en situation de dépendance. Cette dépendance constitue cependant un recours pour exercer ou poursuivre leur métier. De ce fait, la plupart ont une identité qui leur est imposée de l'extérieur par les intégrateurs, les responsables professionnels ou les organismes du développement, qui les ignorent ostensiblement ou dénient leur qualité d'éleveurs. Leur statut est au mieux considéré comme un accès détourné pour exercer leur métier, au pire le produit de leur déchéance du métier d'éleveur. Ce qu'ils mettent en avant est d'abord la dépendance de leur emploi et de leur travail. Dépendance liée à l'évolution des textes réglementaires qui a conduit les naisseurs-engraisseurs à se décharger en partie sur eux, pour se "mettre aux normes ». Dépendance envers leur intégrateur, qui les a conduits à minorer leur engagement dans ce travail, en ne se reconnaissant pas de compétences particulières. Les termes qu'ils emploient pour le qualifier mettent en effet en évidence son peu de valeur à leurs yeux.

Avec les problèmes d'environnement, c'était l'époque où il y a eu des porcheries d'engraissement en façonnage qui ont poussé un peu partout, un peu n'importe comment ... Je suis là juste pour m'en occuper, je les ai trois à quatre mois. Je suis payé ; j'ai cette rémunération de gardiennage. Enfin c'est que dalle l'engraissement ... On gère rien, en fait. On loue de la main-d'œuvre et des bâtiments, c'est tout. (Un éleveur intégré des Côtes d'Armor). 
Cette auto-disqualification est plus accentuée encore quand l'intégration a été un choix contraint de l'éleveur, après une faillite. Pour lui, ce statut est une conséquence de sa déchéance d'éleveur «indépendant ». D'un autre côté cependant, l'intégration constitue aussi, vis-à-vis du regard de ceux qui partagent le territoire qui l'a vu naître, un moyen de masquer son infortune, autant que de conserver son logement. Mais ce masque souligne à quel point l'estime de soi d'un tel éleveur a été mise à l'épreuve.

Moi je travaille là-bas. Il (son intégrateur) fait un peu de façonnage ici et je travaille toujours mes terres. C'est moi qui fais tout le suivi des terres ici avec son matériel. Donc je me retrouve aujourd'hui agriculteur à façon et salarié ... . Dans un premier temps, je m'étais dit : pour préserver ça, un peu de façonnage, ça faisait un complément de salaire. Pourquoi pas. La paille, je garde une vingtaine d'hectares pour moi. Je me suis arrangé avec mon collègue, enfin le patron ... Il y avait le salaire et puis, ici, je pouvais faire un peu de façonnage et le lisier partait sur le terrain. On continuait ; personne ne voyait rien. (Un éleveur de porcs intégré du Finistère).

La carence de reconnaissance identitaire des éleveurs intégrés n'est pas liée qu'aux remarques d'autrui. La reconnaissance du travail n'est pas seule en question, celle du produit l'est aussi. Pour les éleveurs bio de volaille, qui considèrent élaborer un produit de qualité distinctive, l'organisation des filières d'intégration ne permet pas cette rétribution identitaire. En effet, contrairement à ceux qui vendent en filière courte, ils ne peuvent recevoir les commentaires des consommateurs-acheteurs qui, à d'autres, disent apprécier la beauté et la saveur de leurs produits. Au sein même des éleveurs bio, le déni de reconnaissance de ceux qui sont intégrés est également fréquent. L'argument invoqué est l'exigence d'autonomie à laquelle sont attachés la plupart des bio et que réclament leurs cahiers des charges, malgré les dérogations prévues. Alors l'éleveuse dénoncée, pour sa dépendance d'un intégrateur, dénigre en retour ses pairs travaillant en " hors-sol », parce qu'elles manquent manifestement de surface pour nourrir leurs animaux. La disqualification des uns entraîne celle des autres : une manière de se distinguer quelque peu pour certains.

Il y a toujours eu un certain antagonisme entre les gens du GAB et les gens qui faisaient de la pondeuse ... C'est la question de l'intégration ... Aucune banque ne prêtera pour faire un élevage de pondeuse si on n'a pas un contrat d'intégration derrière ... Il y a deux éleveurs qui font de la pondeuse mais c'est de la pondeuse sans rien. Il y a le bâtiment, il y a la pondeuse dedans et ça s'arrête là ... Il y a aussi tout ce côté-là qui est mal vu ... Elles font faire l'épandage à l'extérieur et puis voilà ... Il n'y a 
pas d'implication dans le métier d'agriculteur, c'est ça qui est un peu gênant (une éleveuse de poules pondeuses bio néorurale, intégrée des Côtes d'Armor).

Les éleveurs intégrés se considèrent en situation de dépendance dans l'exercice de leur métier. Certains y ont accédé à la suite d'échecs professionnels tandis que pour d'autres, au contraire, ce fut une opportunité pour accéder au métier. Tous éprouvent cependant des difficultés à faire reconnaître leur travail, du moins en ce qui concerne sa partie sous statut d'intégration. C'est aussi que la plupart le dénigrent eux-mêmes. Ils s'auto-disqualifient, expression manifeste de l'intériorisation de leur statut de population dominée (Paugam, 1991, op. cit).

\subsection{La disqualification par les intégrateurs}

Du côté des intégrateurs, le travail et le métier des éleveurs intégrés sont massivement disqualifiés, voire déniés. Pour la plupart, c'est d'abord le statut et la « responsabilité » qui distinguent, selon eux, qui est éleveur et ne l'est pas. Ce que mettent en relief les propos tenant à distance les anciens éleveurs déchus. L'absence de spécialisation est ici qualifiée de "travail à côté " par les intégrateurs, pour déconsidérer l'éleveur. Ce serait l'envers d'un véritable travail, parce que supportant peu de contraintes. Mais ce travail « à côté » n'est ni un travail rêvé, ni un travail pour soi et les siens, comme l'est le bricolage ou le jardinage ouvrier des années 1970 (Weber, 1989). C'est une disqualification, voire un déni de la qualité du travail qui sont formulés ainsi ; jusqu’à parfois se transformer en rancœur ou en mépris. En revanche, il faut souligner que le travail d'engraissement en élevage de porcs peut être valorisé. C'est le cas lorsqu'un éleveur d'une " grosse structure », en est responsable. Là, le statut prime. Personne ne remet en cause que cet exercice du métier réclame des compétences particulièrement fines de la part de celui qui l'exerce. En effet, d'un point de vue économique, ce poste est stratégique. C'est celui qu'occupe fréquemment alors le responsable de l'élevage, du fait d'une exigence de présence de travail qui peut être fortement limitée par l'automatisation de l'alimentation. Alors, que la durée et la pénibilité du travail y soient limitées n'entache pas la reconnaissance sociale.

C'est la finalité entre tout ce qui est en culture et tout le travail difficile du naissage. Si le gars qui est à l'engraissement n'est pas bon, c'est la tuile ... C'est plus cool ... Physiquement, c'est moins dur. (un éleveur de porcs intégrateur des Côtes d'Armor).

Ce qui domine est la rareté des éleveurs qui reconnaissent le travail des éleveurs intégrés. Ils ne les considèrent bien évidemment pas comme des pairs. Cette conception de ne pas exercer le même métier et de ne pas subir les 
mêmes épreuves à l'occasion de son travail est d'ailleurs partagée. Dépendants et n'assurant qu'une marge du métier, ces façonniers ne peuvent qu'en être rejetés au-delà de la frontière. Ils sont en quelque sorte une dérive voire une dégradation du métier, notamment lorsque ce sont d'anciens éleveurs, ayant abandonné ou été exclus du métier. Leur situation, produit d'un échec, signe leur déchéance.

Sur la région Bretagne, c'est essentiellement des gens qui sont metteurs en pension que l'on trouve, ce n'est pas des donneurs d'ordre. Il y a plus d'éleveurs qui prennent en pension que d'éleveurs qui mettent en pension ... Les gens ont deux, trois façonniers ... Le façonnier, il a ses porcheries, il les a mises à disposition de quelqu'un d'autre et ce n'est plus un entrepreneur ... En général, ce sont des anciens éleveurs qui ont arrêté les truies et qui sont devenus façonniers, ou des anciens engraisseurs qui n'ont pas voulu continuer à prendre des risques ... Il n'est plus éleveur de porcs, il est prestataire de services. La grosse masse des porcs à façon bretons, ce sont des anciens éleveurs ou des anciens engraisseurs, mais pas des gens qui ont investi pour ça spécialement. (un éleveur de porcs intégrateur des Côtes d'Armor).

Les éleveurs intégrés admettent que leur travail est relativement aisé et peu prenant. C'est pour eux un travail dont l'intérêt professionnel et le questionnement sont limités, comparés à ceux de leur engagement professionnel principal. Il n'empêche qu'ils considèrent le bien faire : ce n'est pas pour eux du sale boulot. Cette qualification est un point de vue imposé par leurs intégrateurs et d'autres éleveurs. Aussi leurs relations peuvent-elles être tendues. Cette tension est notamment à l'initiative des éleveurs intégrateurs, qui se sentent obligés de passer par eux et ainsi de perdre en rentabilité. Leur rancour s'exprime en propos dénigrants ... La tension est aussi liée au fait que l'éleveur intégré peut être un ancien naisseur-engraisseur, ce qui réactualise la peur de son intégrateur de déchoir dans un monde concurrentiel, marqué par de multiples exemples de pairs ayant fait faillite. Resurgit alors le spectre du paysan devenu ouvrier. Ayant perdu son «autonomie » et son statut de chef d'entreprise, c'est un déclassé. L'éleveur intégré, dénué de liberté et de créativité, serait ainsi l'envers de la figure de l'entrepreneur, proposé comme modèle à l'éleveur porcin. Dès lors, son travail ne semble plus avoir pour valeur que le revenu complémentaire régulier qu'il lui apporte.

La dévalorisation du travail des éleveurs intégrés permet de souligner en contrepoint les contraintes et les exigences du « naissage ", justifiant en cela la domination de leurs intégrateurs. Les relations qu'entretiennent ceux-ci avec leurs façonniers sont d'ailleurs souvent empreintes de méfiance. Lorsqu'ils évoquent ceux qui travaillent pour eux, leurs commentaires peuvent être 
acerbes. Face aux tensions qui risquent de se manifester, ils ont tendance à les mettre à distance, en recourant à l'intermédiaire de coopératives.

Ce sont souvent les techniciens de coopératives qui font les rabatteurs ... Donc tout se sait ... C'est souvent des situations d'échec, on estime que certains sont un peu des arnaqueurs aussi ... Ce qui fait que, dans de nombreux cas aussi, il n'y a même plus de relations entre le prestataire et le propriétaire des animaux. C'est par le biais de la coop que ça transite. Ce qui fait que ça évite à celui qui met les animaux en place d'avoir des relations à nouer. Tout est mathématique. Et en fait les basses besognes sont réalisées par la coop. C'est confortable en termes de relations ! (Un éleveur de porcs des Côtes d'Armor, intégrateur).

Certains intégrateurs dénient autant le métier d'éleveurs à ceux qui sont intégrés que leur implication dans leur travail. Pour eux, ils ne sont qu'exécutants de leurs prescriptions. C'est pourquoi ils se sentent autorisés à considérer que ces éleveurs seraient dans des situations privilégiées, parce qu'ayant peu de compétences à exercer. Tandis qu'eux effectueraient le travail le plus délicat et assumeraient tous les risques économiques. Cependant, cette dénégation du métier des éleveurs intégrés est partagée. Dans la relation entre donneur d'ordre et façonnier se manifestent des rapports de domination, qui conduisent à disqualifier le travail effectué, jusqu'à le rendre impur, parce qu'entaché par la déchéance de certains, et invisible, parce que socialement ignoré. Plus largement, les éleveurs intégrés comme les intégrateurs, au travers des termes qu'ils emploient pour caractériser ce métier et le travail qu'il recouvre, s'accordent pour le dévaloriser.

\section{Conclusion}

Le statut des éleveurs intégrés recouvre des situations contrastées. Selon les filières, la relation d'intégration differe. En porc, ce sont aussi bien des éleveurs naisseurs-engraisseurs que des groupements de producteurs de firmes - privées ou coopératives - qui intègrent les éleveurs. En volaille et en veau, seuls les groupements de producteurs interviennent. Dans le premier cas, l'absence de pairs des éleveurs limite leur reconnaissance sociale. La relation d'intégration oblige cependant à une dépendance mutuelle intégré-intégrateur : entre défiance dans certains cas, confiance dans d'autres. Certes, la dimension hiérarchique et prescriptive de la relation suscite des tensions. Elles sont cependant tempérées, car les intégrateurs porcins apprécient outre les problèmes d'épandage de lisier qu'ils leur résolvent, qu'ils leur permettent de valoriser l'ensemble de leur production, tout en réduisant leur coût alimentaire, par l'achat global de l'alimentation de l'ensemble de 
leurs animaux. Ces intégrateurs se créent également ainsi un réseau d'obligés, en offrant des emplois. Lorsque la relation implique des firmes, les éleveurs peuvent être l'objet d'un mépris plus ou moins affiché. Il est accentué envers les femmes, dans ce secteur où le référentiel du métier est masculin (Rieu et Dahache, 2008). D’autres éleveurs intégrés s'impliquent avec leurs pairs au sein du Groupement de Producteurs de leurs firmes intégratrices. Alors, leurs réunions techniques et le débat des manières de travailler de chacun suscitent des jugements, producteurs de reconnaissance du travail, alimentant l'identité de chacun. La dynamique peut être d'autant plus importante lorsque l'entreprise joue de sa notoriété, pour chercher à construire ainsi une culture d'entreprise : des valeurs reliées à des manières de faire initiées par l'entreprise, qu'adoptent peu ou prou ceux qui en dépendent. Ainsi certaines multiplient-elles les réunions d'éleveurs, en offrant du conseil et de l'aide, au-delà du domaine délimité par le contrat d'intégration. Cette culture peut aussi se traduire, par exemple en volaille, par le respect des animaux que suscite une règle implicite partagée : passer du temps pour leur apprendre à sortir, ce qui amène à se démarquer d'autres intégrateurs ... Dans la plupart des cas cependant, l'intégration par la firme se distingue globalement de celle de l'éleveur intégrateur. Que ce soit en porc, en veaux ou en volaille, les techniciens de la firme exercent une relation souvent distanciée, dénuée de rapports de sociabilité. Les relations sont alors formalisées et même souvent réduites au débat des résultats chiffrés, ce qui tend à les déshumaniser (Schwartz, 2000).

Comme en milieu industriel, l'intégration est une voie de modernisation pour les petites entreprises. Certaines ont ainsi accès à une assistance technique comme à des prêts qu'elles ne trouveraient pas ailleurs. Plus largement, l'intégration limite leur disparition (Houssiaux, 1957, 2). Comme pour les sous-traitants de l'industrie, la contribution des éleveurs intégrés a été de limiter les effets des évolutions et des fluctuations de la production de ceux qui les dominaient, au risque de leur propre précarisation. Cependant le parcours professionnel de ces éleveurs, qui les désigne souvent comme des rescapés ou des marginaux de l'agriculture, suscite une disqualification de leur travail et un déni de reconnaissance de leurs manières d'exercer leur métier. Porteurs de cette disqualification, leurs intégrateurs ne les reconnaissent pas pour pairs, parce que dépendants. Même si on peut s'interroger, avec Guiqueno et Daucé (1984), sur la différence d'autonomie dans le travail entre ces éleveurs et leurs intégrateurs "indépendants ", en contrat avec leurs groupements de producteurs et plus largement avec l'aval de leur filière. En revanche, la plupart des éleveurs intégrés ont un travail «à côté ", dans lequel ils donnent toute la mesure de leurs compétences d'agriculteurs. Cet «à côté » est une bouffée identitaire qui leur permet de "tenir », face au déni de leur métier. De même que le racisme peut se cumuler au mépris social pour occulter l'existence et le travail de femmes de ménage, au point que leur présence physique soit niée socialement (Honneth, 2004), la disqualification sociale des éleveurs intégrés les rend, comme leur travail, invisibles. C'est moins de 
leur travail que de leur statut dont il s'agit : c'est de leur position sociale au sein d'un autre monde : celui de l'élevage. À ce niveau, ils subissent la cascade de petits mépris qui recoupent les hiérarchies instituées des professions. Un tel jugement d'autrui alimente également celui de soi (Hugues, 1996). Leur disqualification sociale se redouble d'une auto-disqualification, qui les affecte d'autant plus que leurs relations entre pairs sont rares. Cette rareté ne permet pas en effet leur confrontation : celle de leurs manières de travailler, des difficultés qu'ils éprouvent et des prouesses qu'ils réalisent dans l'exercice de leur métier, qui confortent l'identité de chacun. À l'instar de ce que montre Paugam (2007) des salariés précarisés, le statut dégradé, la vulnérabilité des situations d'emploi et le manque d'échanges entre éleveurs intégrés limitent leur reconnaissance. C'est pourquoi certains mettent en avant le travail dans lequel ils s'investissent. Ce qui toutefois ne les empêche ni de souffrir, ni de considérer bien faire leur travail d'éleveurs intégrés. Même s'ils savent qu'ils ne seront pas reconnus à son propos. Au final se retrouve, au travers de la relation d'intégration, une distinction $\mathrm{du}$ « bon travail » et du « sale boulot »: ces tâches jugées dégradantes par les membres d'un groupe au travail et qui estiment qu'elles remettent en cause leur statut professionnel (Hughes, 1996, op. cit). Dès lors, les parcours d'accès des éleveurs et les conditions d'exercice de leurs tâches éclairent les multiples tensions qui traversent l'organisation des filières d'intégration.

\section{Références bibliographiques}

Baudry B. (1994) De la confiance dans la relation d'emploi ou de sous-traitance, Sociologie du travail, 36(1), 43-61.

Bodiguel M., Jollivet M., et Mainié P. (1971) Structures agraires et diffusion de l'économie contractuelle en agriculture, CNRS, groupe de recherches sociologiques de l'Université de Paris X (Nanterre), M. Jollivet (dir.), t. 4, Synthèse et conclusion, Paris : Commissariat du Plan, CORDES, DGRST, Comité socio-économie du développement, $422 \mathrm{p}$.

Bonnaud M. L., Nicourt C. (2006) Les éleveurs de porcs face à leurs détracteurs en Dordogne et dans le Finistère, Études Rurales, 177(1), 55-68.

Burguière A. (1975) Bretons de Plozevet, Flammarion, Paris, 383 p.

Callais M.-J., Pendaries C. (2010) Les exploitations agricoles en 2008. Les volailles de qualité marquent le pas, Agreste Primeur, 238, avril, 4 p.

Canguilhem G. (1966) Le normal et le pathologique, Paris, PUF, "Quadrige », réédition 1993, $232 \mathrm{p}$.

Castel R. (1991) De l'indigence à l'exclusion, la désaffiliation. Précarité du travail et vulnérabilité relationnelle, in : «Face à l'exclusion. Le modèle français ». Donzelot J. (dir.), Esprit, 137-168. 
Coléou J. (1997) L'apparition des modes intensifs en élevage, in : « Le mangeur et l'animal. Mutations de l'élevage et de la consommation », Paillat M. (dir.), Autrement, 172, 42-68.

Colson F. (1980) Modernisation de la production porcine : les limites économiques au processus de rationalisation de l'élevage porcin, Journées de la Recherche Porcine, 6 et 7 février, 59-71.

Corbin A. (1982) Le miasme et la jonquille. L'odorat et l'imaginaire social XVIII XIX ${ }^{\mathrm{e}}$ siècles, Paris, Aubier-Montaigne, 334 p.

Cornu G. (1987) Vocabulaire juridique, Paris, PUF, 839 p.

Davezies P. (1993) Éléments de psychodynamique du travail, Éducation Permanente, 116, 33-46.

Dejours C. (2000) Travail, usure mentale, Paris, Bayard, $3^{\mathrm{e}}$ ed., 281p.

Diry J. P. (1985) L'industrialisation de l'élevage en France. Économie et géographie des filières agricoles et porcines, Paris, Ophrys, 680 p.

Dobry M. (2000) Les voies incertaines de la transitologie. Choix stratégiques, séquences historiques, bifurcations et processus de path dependence, Revue française de science politique, 50(4-5), 585-614.

Ehrenberg A. (1991) Le culte de la performance, Paris, Calmann-Levy, 323 p.

Ehrenberg A. (2010) La société du malaise. Le mental et le social, Paris, Odile Jacob, 439 p.

Estrangin L. (1962) Du paysan à l'agriculteur, Paysans, 28-37.

Guiqueno L., Daucé P. (1984) Aux origines de la modernisation agricole et de l'intensification de l'agriculture en Bretagne, Norois, 124, 541-557.

Honneth A. (2004) Visibilité et invisibilité. Sur l'épistémologie de la « reconnaissance ", Revue du MAUSS, 23(1), 137-151.

Houssiaux J. (1957) 1- Le concept de « quasi-intégration » et le rôle des soustraitants dans l'industrie, Revue économique, 8(2), 221-247.

Houssiaux J. (1957) 2- Quasi-intégration, croissance des firmes et structures industrielles, Revue économique, 8(3), 385-411.

Hughes E., C. (1996) Le regard sociologique. Essais choisis, Paris, Éd. de l’EHESS, $344 \mathrm{p}$.

Joly N. (2009) Écrire l'événement : le travail agricole mis en mémoire, Sociologie du Travail, 46(4), 511-527.

Keyser V. de, Olivier M. (1972) Le besoin de cohérence : dimension sociale et individuelle du champ cognitif de l'homme au travail, Le Travail Humain, 35(1), 49-58.

Lacombe P. (1968) Réflexions sur l'agriculture à temps partiel, Économie Rurale, 76, 91-99. 
Lamarche H. (1971) La région centre du Morbihan : région de St Jean-Brevelay, in : Structures agraires et diffusion de l'économie contractuelle en agriculture, CNRS, groupe de recherches sociologiques de l'Université de Paris X (Nanterre), M. Jollivet (dir.), t. 4, Synthèse et conclusion, Paris : Commissariat du Plan, CORDES, DGRST, Comité socio-économie du développement, $232 \mathrm{p}$.

Latreille G. (1979) Naissance des métiers et professions dans la France contemporaine, Thèse, Université de Paris V. Lyon, Presses universitaires de Lyon, 408 p.

Lequin Y. (1977) Les ouvriers de la région lyonnaise, 1848-1914, Presses Universitaires de Lyon, 2 vol, 1073 p.

Liaudon M. (1964) Pour une agriculture de groupe, industrielle et commerciale, rapport au $9^{e}$ congrès du CNJA (Centre national jeunes agriculteurs), Paris, SEPJA, 71 p.

Maget M. (1955) Remarques sur le village comme cadre de recherches anthropologiques, Bulletin de psychologie, 8(7-8), 375-382.

Malassis L. (1964) Essai d'orientation sur l'évolution de la coopération en France, Économie rurale, 62, 25-33.

Mayaud J.-L. (1999) De la pluri-activité paysanne à une re-définition de la petite exploitation rurale, Annales de Bretagne et des pays de l'Ouest, 106(1), 231-248.

Montmollin M., de (1983) L'intelligence de la tâche, éléments d'ergonomie cognitive, Berne, Peter Lang, 187 p.

Nicourt C. (1999) Entre archaïsme et modernité, le coût humain du travail des agricultrices dans les exploitations familiales, Le Travail Humain, 62(2), 155-171.

Nicourt C., Filippi G. (1987) Contribution à la définition d'un métier : agricultrice. Sociologie du Travail, 29(4), 477-494.

Nicourt C., Girault J. M. (2011) La normalisation du travail viticole à l'épreuve de l'usage des pesticides, Économie Rurale, 1, 321, 29-41.

Osty F. (2003) Le désir du métier : engagement, identité et reconnaissance au travail, Rennes, PUR, 244 p.

Paugam S. (1991) La disqualification sociale : Essai sur la nouvelle pauvreté, Paris, PUF, $254 \mathrm{p}$.

Paugam S. (2007) Le salarié de la précarité : Les nouvelles formes de l'intégration professionnelle, Paris, PUF, 437 p.

Rambaud P. (1973) Les coopératives de travail agraire en France, Paris, EPHECentre de sociologie rurale, $166 \mathrm{p}$. 
Rémy J. (1971) Le Soissonnais, in : Structures agraires et diffusion de l'économie contractuelle en agriculture, CNRS, groupe de recherches sociologiques de l'Université de Paris X (Nanterre), M. Jollivet (dir.), t. 3, Synthèse et conclusion, Paris: Commissariat du Plan, CORDES, DGRST, Comité socio-économie du développement, 232 p.

Paysan Revue (1961).

Rieu A., Dahache S. (2008) S'installer comme agricultrice : sur la socialisation et la formation sexuée en agriculture, Revue d'Études en Agriculture et Environnement, 88(3), 71-94.

SCEES (2004) $14 \%$ de porcs à l'engraissement en pension. Agreste Primeur, $148,4 \mathrm{p}$.

Schaller B. (1979) L'aviculture française vingt ans après : les limites de la « révolution avicole » dans la filière volailles de chair, Économie rurale, $132,57-63$.

Schwartz Y. (2000) Le paradigme ergologique ou le métier de philosophe, Toulouse, Octarès, 763 p.

Segrestin D. (1996) La normalisation de la qualité et l'évolution de la relation de production, Revue d'économie industrielle, 75, 291-307.

Shaw G. (1981) Le travail des femmes. Éléments pour une recherche ergonomique, DEA d'ergonomie, CNAM-Université Paris XIII, 71 p.

Terssac G. de (1992) Autonomie dans le travail, Paris, PUF, 288 p.

Vennin B. (1975) Pratique et signification de la sous-traitance dans l'industrie automobile en France, Revue Économique, 26(2), 280-306.

Wackermann G. (1977) La place des foires et marchés parmi les pôles français de commercialisation des produits agricoles, Économie Rurale, 122, 8-22.

Weber F. (1989) Le travail à-côté : étude d'ethnographie ouvrière, Paris, EHESS et INRA, 207 p. 
